\title{
Comparative sensitivity evaluation for 122 CE-marked SARS-CoV-2 antigen rapid tests
}

Heinrich Scheiblauer ${ }^{1}$, Angela Filomena ${ }^{1}$, Andreas Nitsche ${ }^{2}$, Andreas Puyskens ${ }^{2}$, Victor M Corman ${ }^{3}$, Christian Drosten ${ }^{3}$, Katrin Zwirglmaier ${ }^{4}$, Constanze Lange ${ }^{5}$, Petra Emmerich $^{6}$, Michael Müller ${ }^{7}$, Olivia Knauer ${ }^{1}$, C Micha Nübling $^{1^{*}}$

${ }^{1}$ Paul-Ehrlich-Institute, Paul-Ehrlich-Str. 51-59, D-63225 Langen

${ }^{2}$ Robert Koch-Institute, Seestrasse 10, D-13353 Berlin

${ }^{3}$ Institute of Virology, Charite, Chariteplatz 1, D-10117 Berlin

${ }^{4}$ Bundeswehr Institute of Microbiology, Neuherbergstr 11, D-80937 Munich

${ }^{5}$ LADR GmbH, Lauenburger Str. 67, D-21502 Geesthacht

${ }^{6}$ Bernhard-Nocht Institute, Dep.Virology, Bernhard-Nocht Str. 74, D-20359 Hamburg

${ }^{7}$ MVZLabor $28 \mathrm{GmbH}$, Mecklenburgische Str. 2, D-14197 Berlin

*Corresponding Author: PD Dr C Micha Nübling

Paul-Ehrlich-Institut (PEI)

Paul-Ehrlich-Str. 51-59, 63225 Langen, Germany

Phone: +49 6103 773304; Email: micha.nuebling@pei.de

This study has been submitted as tandem manuscript together with the study of Puyskens A et al. "Establishment of an evaluation panel for the decentralized technical evaluation of the sensitivity of 31 rapid detection tests for SARS-CoV-2 diagnostics" 


\begin{abstract}
Objective

Independent evaluation of the sensitivity of CE-marked SARS-CoV-2 antigen rapid diagnostic tests (Ag RDT) offered in Germany.
\end{abstract}

\title{
Method
}

The sensitivity of $122 \mathrm{Ag}$ RDT was adressed using a common evaluation panel.

Minimum sensitivity of $75 \%$ for panel members with $\mathrm{CT}<25$ was used for differentiation of devices eligible for reimbursement in in the German healthcare system.

\section{$\underline{\text { Results }}$}

The sensitivity of different SARS-CoV-2 Ag RDT varied over a wide range. The sensitivity limit of $75 \%$ for panel members with CT <25 was met by 96 of the 122 tests evaluated; 26 tests exhibited lower sensitivity, few of which were completely failing. Some devices exhibited high sensitivity, e.g. 100\% for $\mathrm{CT}<30$.

\section{Conclusion}

This comparative evaluation succeeded to distinguish less sensitive from better performing Ag RDT. Most of the Ag RDT evaluated appear to be suitable for fast identification of acute infections associated with high viral loads. Market access of SARS-CoV-2 Ag RDT should be based on minimal requirements for sensitivity and specificity. 
medRxiv preprint doi: https://doi.org/10.1101/2021.05.11.21257016; this version posted May 12, 2021. The copyright holder for this preprint (which was not certified by peer review) is the author/funder, who has granted medRxiv a license to display the preprint in perpetuity.

All rights reserved. No reuse allowed without permission.

\section{Introduction}

A large number of antigen-detecting rapid diagnostic tests (Ag RDTs) for SARS-CoV2 are available on the European market, both for professional use and as self-tests. Rapid tests are based on lateral flow immunochromatography using antibodies against SARS-CoV-2 proteins (antigens), present in respiratory tract specimens. By far most Ag RDTs target the viral nucleoprotein $(\mathrm{N})$, only very few assays work with spike protein (S) detection. Viral variants of concern (VOC) have been described mainly for the S gene, leaving the vast majority of SARS-CoV-2 Ag RDT unaffected; however, the few SARS-CoV-2 Ag RDT based on spike protein detection should be checked at regular intervals for potential deficiencies. While PCR is still the gold standard for virus detection, there is an increasing evidence that infectivity by respiratory secretions correlates with high viral loads present in the early phase of infection, e.g. before and after (0-10 days) onset of symptoms. Thus Ag RDTs allow rapid identification of acutely infected and potentially infectious individuals facilitating fast decisions on containment of virus spread, patient care, isolation and contact tracing. Furthermore, Ag RDTs may save limited reagents of more sensitive molecular diagnostics to serve other diagnostic needs, e.g. disease management or confirmation of $\mathrm{Ag}$ RDT reactive results.

In the European Union (EU), regulatory requirements for SARS-CoV-2 in vitro diagnostic medical devices (IVD) are defined by the IVD Directive 98/79/EC (IVDD) and have to be addressed by the manufacturer prior to access to the EU Common Market. However, certification (CE-marking) of SARS-CoV-2 diagnostics is currently done solely by the manufacturer (self-certification), without third party intervention. The exception are SARS-CoV-2 self-tests, where a notified body has to assess the lay person studies. However, due to the urgency in the Corona situation a national derogation can be agreed by the national Competent Authority, e. g. based on the performance of an identical test for professional use. From May 2022 the IVDD will be replaced by the IVD Regulation (EU) 2017/746 (IVDR) where a risk-based classification of IVDs is the basis for the scrutiny of their assessment (1). SARS-CoV2 IVD will belong to the high-risk devices (class D) under the IVDR, requiring a Notified Body both for certification of the manufacturer's quality management system and for assessment of the technical documentation of the device. Furthermore, EU reference laboratories (EURL) will be responsible for independent laboratory testing 
medRxiv preprint doi: https://doi.org/10.1101/2021.05.11.21257016; this version posted May 12, 2021. The copyright holder for this preprint (which was not certified by peer review) is the author/funder, who has granted medRxiv a license to display the preprint in perpetuity.

All rights reserved. No reuse allowed without permission.

of class $D$ devices to verify performance features and to assure batch to batch consistency.

However, at the time being independent evaluations of SARS-CoV-2 Ag RDT that allow conclusions on their performance are largely missing.

In the current situation with absence of strict regulatory requirements for most SARSCoV-2 IVD, the German Ministry of Health decided to link the reimbursement of SARS-CoV-2 Ag RDT to provision of evidence of essential quality features of these assays. This evidence consisted of two parts: 1) provision by the manufacturer of evidence for compliance with minimum criteria, and 2) successful independent laboratory evaluation. Minimum criteria for sensitivity and specificity were jointly defined by Paul-Ehrlich-Institut (PEI) and Robert Koch Institut (RKI), two governmental authorities in Germany (2). Manufacturers or distributors of SARSCoV-2 Ag RDT document for the respective SARS-CoV-2 Ag RDT compliance with these minimum criteria before the device can be listed as eligible for reimbursement on a dedicated webpage of Bundesinstitut für Arzneimittel und Medizinprodukte (BfArM), another governmental authority (3).

Devices were selected from the BfArM list for the comparative evaluation performed by $\mathrm{PEI} / \mathrm{RKI}$. The aim of this comparative evaluation was to both determine the "state of art" sensitivity of proficient devices and identify devices not reaching the minimum sensitivity level. Subsequently, devices with sensitivity below "state of the art" were removed from the BfArM list while all devices with successful evaluation outcome were published on PEI webpage (4). In the meantime more than 120 SARS-CoV-2 Ag-RDT have been evaluated in direct comparison using common SARS-CoV-2 specimens. The outcome and conclusions of this study are summarized in this manuscript. 
medRxiv preprint doi: https://doi.org/10.1101/2021.05.11.21257016; this version posted May 12, 2021. The copyright holder for this preprint (which was not certified by peer review) is the author/funder, who has granted medRxiv a license to display the preprint in perpetuity.

All rights reserved. No reuse allowed without permission.

\section{Materials and Methods}

\section{Evaluation panel}

Detailed characterization of the evaluation panel has been described by Puyskens et al. in the tandem publication to this study. In short, pools from nasopharyngeal and oropharyngeal swabs were prepared as random mixtures obtained from up to 10 swabs. While dry swabs were directly eluted in phosphate buffered saline (PBS), the residual amount virus transport media (VTM) contained in moist swabs was diluted in PBS. Care was taken not to use VTM containing the protein denaturing component guanidinium.

Individual pools are composed of samples with similar SARS-CoV-2 concentrations, expressed as cycle threshold (CT) values of semiquantitative PCR. In total 50 different pools were defined as members of the evaluation panel and stored as $500 \mu \mathrm{l}$ aliquots at $-80^{\circ} \mathrm{C}$. The CT of each panel member was determined by PCR, and the putative number of RNA copies calculated with the aid of the reference preparations distributed by the German external quality assessment (EQA) provider INSTAND e. V. (5). Furthermore, presence of infectious virus detectable by propagation in Vero cell culture was determined for the individual pools.

The whole evaluation panel may be subdivided into three subgroups: panel members, which are characterized by very high (CT 17-25; 18 pools), high (CT 2530; 23 pools) or moderate (CT 30-36; 9 pools) viral load. During the comparative evaluation 4 panel members of the original panel had to be replaced, resulting in a slight shift of subgroups composition in the resulting panel version 2: 17 pools covering the CT-range 17-25, 23 pools the CT range $25-30$ and 10 pools the CT range 30-36.

\section{Antigen stability}

Real-time antigen stability in panel members was investigated using quantitative SARS-CoV-2 ELISA Lumipulse G SARS-CoV-2 Ag (Fujirebio Inc., Shinjuku-ku, Tokyo, Japan). Panel members were tested after initial thawing and throughout one week incubation at $4^{\circ} \mathrm{C}$. Furthermore, potential impact of additional freeze/thaw cycle was addressed. 
medRxiv preprint doi: https://doi.org/10.1101/2021.05.11.21257016; this version posted May 12, 2021. The copyright holder for this preprint (which was not certified by peer review) is the author/funder, who has granted medRxiv a license to display the preprint in perpetuity.

All rights reserved. No reuse allowed without permission.

\section{Comparative evaluation}

In the beginning of the comparative evaluation, laboratories participating in the comparative evaluation included the Robert Koch-Institute, the Paul-Ehrlich-Institute, the Nationales Konsiliarlaboratorium für Coronaviren (Charité), the Bundeswehr Institute of Microbiology, the Bernhard-Nocht-Institut für Tropenmedizin, and laboratories of the association ALM (Akkreditierte Labors in der Medizin). At a later stage, because of the massively increasing work load, the evaluation was continued mainly by PEI and RKI. Panels were shipped on dry ice and, once thawed, $50 \mu \mathrm{l}$ aliquots were prepared, kept at $4^{\circ} \mathrm{C}$ and used within 5 days, without further freeze / thaw step. For each Ag RDT and panel member, the $50 \mu$ aliquot was completely absorbed using the specimen collection device, e.g. swab, provided with the respective test. The swabs were then eluted in the test-specific buffer, strictly following the respective instructions for use (IFU). After applying the sample/buffer solution onto the test cassette and incubation, visual read out of control and target lines was done independently by two lab technicians, with potential discrepant results preliminarily interpreted as "equivocal". In favor of the tests evaluated, both reactive and equivocal results for the target line were eventually scored as positive. At PEI the test cassettes were immediately scanned using BLOTrix Reader R2L (BioSciTec $\mathrm{GmbH}$ ) and analysed with BLOTrix 4 Cubos (B4C) software (BioSciTec $\mathrm{GmbH}$ ), at other evaluation sites the test results were documented by photographs. Some tests were provided with reading instruments and read as per instruction manual provided.

Tests were selected from original manufacturers, as far as this information was available. Often duplicate version of the very same tests are marketed under a new test name, new manufacturer or different distributor. Repeat testing of duplicates was avoided as far as possible in order to cope with the already huge variety of different tests placed on the EU Common Market. 
medRxiv preprint doi: https://doi.org/10.1101/2021.05.11.21257016; this version posted May 12, 2021. The copyright holder for this preprint (which was not certified by peer review) is the author/funder, who has granted medRxiv a license to display the preprint in perpetuity.

All rights reserved. No reuse allowed without permission.

\section{Results}

\section{Characterization of the evaluation panel}

Panel members spanned the CT range between 17 and 36. A specimen with an assigned SARS-CoV-2 RNA concentration of $10^{6}$ RNA copies $/ \mathrm{ml}$ provided by INSTAND corresponded to the CT value of 25. Assuming that a CT difference of 1 corresponds to a concentration factor of 2 and taking into account that the individual panel members cover a CT range from 17 to 36 , the SARS-CoV-2 RNA amounts in the panel cover a concentration range from $>10^{8}$ to $<10^{3}$ copies $/ \mathrm{ml}$, respectively. The CT values of 20 or 30 would than correspond to approximate SARS-CoV-2 RNA concentrations of $3 \times 10^{7}$ or $3 \times 10^{4}$ copies / ml, respectively.

SARS-CoV-2 propagation in cell culture resulted in positive results for several of the low CT / high titre specimens, indicating presence of infectious virus despite the various preparation steps (more details in the tandem publication of Puyskens et al).

Stability of the analyte SARS-CoV-2 antigen in all panel members was studied under different conditions using a quantitative ELISA. While additional freeze/thaw steps had negative effect on analyte stability, there was no significant impact on the antigen content after up to 7 days storage at $4^{\circ} \mathrm{C}$ of the liquid $50 \mu$ aliquots (data not shown). From one $500 \mu$ thawed aliquot, nine to ten $50 \mu$ aliquots were immediately filled and used within 5 days for the corresponding number of 9-10 test kits, ensuring no stability issues.

\section{Comparative evaluation}

122 SARS-CoV-2 rapid tests were evaluated in direct comparison using the evaluation panel, with only few differences between the closely related panel versions 1 and 2. For acceptable Ag RDT performance a minimum sensitivity of $75 \%$ for the panel member subgroup with very high SARS-CoV-2 concentration $(C T<25$, viral load around $10^{6}$ SARS-CoV-2 RNA/ml and higher) was defined. This criterion corresponds to the detection of at least 14 of 18 subgroup positives in panel version 1 (18 members with $\mathrm{CT}<25)$, or 13 of 17 (17 members with $\mathrm{CT}<25$ in panel version 2), respectively. 
medRxiv preprint doi: https://doi.org/10.1101/2021.05.11.21257016; this version posted May 12, 2021. The copyright holder for this preprint (which was not certified by peer review) is the author/funder, who has granted medRxiv a license to display the preprint in perpetuity.

All rights reserved. No reuse allowed without permission.

Of the 122 SARS-CoV-2 Ag RDT evaluated, 96 tests (78.7\%) had a sensitivity of $>75 \%$ for panel members with high viral loads (CT<25; Table 1 ), and 26 tests

$(21.3 \%)$ were of lower sensitivity not meeting the sensitivity criterion (Table 2 ). Of the 96 tests meeting the sensitivity limit, 58 (60.4\%) detected all panel members of the subgroup with CT< 25 (100\% subgroup sensitivity), and another 17 tests $(17.7 \%)$ exhibited a respective subgroup sensitivity of $>90 \%$. In addition, 19 tests (19.8\%) showed a sensitivity of $>75 \%$ even in the CT range $25-30$.

The 96 tests meeting the sensitivity criteria were reactive with between 14 and 41 members of the 50 members panel (supplemental Table 1). On average throughout all successful tests, 27 panel members were reactive. Overall reactivity of SARSCoV-2 Ag RDT strongly followed the analyte concentration throughout the panel, justifying the conclusions of this study (supplemental Table 1).

The 26 SARS-CoV-2 Ag RDT missing the sensitivity criteria either failed completely (2 tests with 0 reactives) or were reactive with 2 to 12 (average 6.3) panel members. Again, reactivity was dependent on the analyte concentration throughout the panel members (supplemental Table 2). Two tests failed because of constant faint background reactivity throughout all panel members; this background reactivity was also seen when using pure extraction buffer and was thus not caused by the panel composition (data not shown). 
medRxiv preprint doi: https://doi.org/10.1101/2021.05.11.21257016; this version posted May 12, 2021. The copyright holder for this preprint (which was not certified by peer review) is the author/funder, who has granted medRxiv a license to display the preprint in perpetuity.

All rights reserved. No reuse allowed without permission.

\section{Discussion}

There is convincing evidence that infectivity of SARS-CoV-2 correlates directly with high viral loads in respiratory specimens of acutely infected persons. It has therefore been suggested to use antigen tests rather to detect potential infectivity to help to control the spread of infection than for the purpose of clinical diagnosis. Ag RDTs are designed to detect SARS-CoV-2 antigens in respective samples and have become a key part of testing strategies in many countries since fall 2020. Hundreds of different Ag RDTs, most often of East-Asian origin, are available on the Common Market in Europe. Nearly all tests state in their IFUs sensitivity values of $>90 \%$ for PCRconfirmed specimens. Such statements are unreliable and in strong contrast to the results of our study and to independent evaluations. Their only explanation is a strong preselection of high-positive specimens.

Lack of independent evaluation combined with unreliable statements of quality features led the German Ministry of Health to request a comparative evaluation of the sensitivity of test kits offered in Germany. At the time being there are no EU-wide requirements for quality features of COVID-19 IVDs such as a defined minimum sensitivity or minimum specificity, and manufacturers may themselves certify their devices to comply with basic requirements of the IVDD. Therefore, it is mainly left to individual Member States or international organizations to define minimum requirements for acceptance of respective tests.

In Germany, the Ministry of Health decided to link the reimbursement of SARS-CoV-2 Ag RDT to quality requirements to be fulfilled by acceptable devices. Minimum requirements were jointly formulated by Paul-Ehrlich-Institute and Robert KochInstitute and state for SARS-CoV-2 Ag RDTs a minimum sensitivity of $80 \%$ for PCR positive specimens obtained within the first seven days after symptom onset; the minimum specificity was defined as $>97 \%$, and for both requirements a study population of at least 100 persons is required. Analogous requirements of SARSCoV-2 Ag RDTs have been proposed by the World Health Organization for the emergency use listing (EUL) (6), the US Food and Drug Administration (FDA) (7), the European Center for Disease Control (ECDC) (8), the Swiss Authority Bundesamt für Gesundheit (BAG) (9) or the non-governmental Foundation for Innovative New Diagnostics (FIND) (10). With mandatory application of the EU IVD Regulation (IVDR) for SARS-CoV-2 Ag RDT their regulatory oversight will improve in near future. 
medRxiv preprint doi: https://doi.org/10.1101/2021.05.11.21257016; this version posted May 12, 2021. The copyright holder for this preprint (which was not certified by peer review) is the author/funder, who has granted medRxiv a license to display the preprint in perpetuity.

All rights reserved. No reuse allowed without permission.

In this context it might be of interest that so-called Common Specifications have already been drafted for SARS-CoV-2 IVD. Although these draft quality requirements are still under consultation and not yet in effect, they will become essential prerequisites for future CE-marking of respective devices.

For the time being, successful participation in a comparative evaluation of the crucial test feature sensitivity was installed as another precondition for reimbursement of SARS-CoV-2 Ag RDT by the German healthcare system. The first round of this evaluation is summarized in this manuscript.

The sensitivity requirement defined for a successful outcome in the comparative evaluation study $(>75 \%$ sensitivity for $\mathrm{CT}<25)$ is in line with international requirements mentioned above, as far as clinical specimens from acutely infected individuals are comparable to the members of our evaluation panel. We followed routine use of the tests as far as possible, including pre-analytical steps like antigen absorption of the test-specific swabs, and subsequent elution into the test-specific buffer. The vast majority of Ag RDTs included in our study showed sufficient sensitivity according to our criteria. Nevertheless, the results showed a wide range of varying sensitivities. There were few tests with fairly high and many tests with sufficient sensitivity, but also quite a few tests, which did not meet the minimum criterion. The study shows that the majority of SARS-CoV-2 Ag RDTs correctly

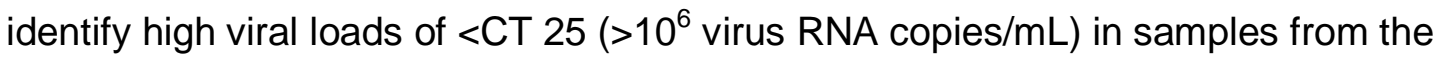
respiratory tract with a sensitivity of $>75 \%$, supporting their use in the early symptomatic phase. However, although sensitivity often declined with $\mathrm{CT}>25$, there were also few SARS-CoV-2 Ag RDTs with quite high sensitivity, even 100\% for $\mathrm{CT}<30$, or up to $86 \%$ for the complete CT range (CT 17-36). There are scientific publications of further independent head-to-head evaluations for SARS-CoV-2 Ag RDT which, however, are limited to the comparison of only few tests (11-17). Respective conclusions based on clinical specimens are consistent with our results, and the sensitivity ranking of different tests was widely in line with our results based on the evaluation panel.

Since most of the SARS-CoV-2 Ag RDTs offered in Europe are provided without a readout device, visual interpretation of test results is indispensable. We would like to emphasize that few discrepant tests results obtained by two experienced lab technicians were reported. These equivocal results were ultimately interpreted as 
reactive, in favor of the tests under investigation. However, visual readout and subjective interpretation of faint test lines, potentially caused by borderline concentration of the analyte, presents a challenge for less experienced users, e.g. lay persons using Ag RDTs as self tests.

A limitation of the study is its spot check nature since it cannot address variations between different batches of the same product, or variations between different test locations (see also the tandem publication of Puyskens et al).

In conclusion, by using the same panel for a large number of different SARS-CoV-2 Ag RDT we were able to evaluate the comparative performance of the different tests under the same conditions. The evaluation panel proved to be accurate for sensitivity differentiation of SARS-CoV-2 Ag RDTs, distinguishing better performing from less suitable tests. The continuation of the comparative evaluation is needed cope with the rapidly growing market of SARS-CoV-2 Ag RDT. Since the panel has now been exhausted, we will continue the evaluation with a new set of samples with similar features, accurately calibrated against its predecessor. 


\section{References}

1) Mbunkah HA, Reinhardt J, Kafere $C$, Scheiblauer $H$, Prat I, Nübling $C M$ (2021) In vitro diagnostics for screening the blood supply: the new European regulation for IVD and the WHO IVD prequalification programme. Vox Sang 116, 3-12. https://doi.org/10.1111/vox.12996

2) Paul-Ehrlich-Institut. Minimum Criteria for Rapid SARS-CoV-2 Antigen Tests. https://www.pei.de/SharedDocs/Downloads/EN/newsroomen/dossiers/minimum-criteria-for-rapid-sars-cov2-antigen-tests-01-122020.pdf?_blob=publicationFile\&v $=5$

3) Bundesinstitut für Arzneimittel und Medizinprodukte. Liste der AntigenTests zum direkten Erregernachweis des Coronavirus SARS-CoV-2. https://antigentest.bfarm.de/ords/f?p=101:100:9913504321339::.:.:\&tz=2:00

4) Paul-Ehrlich-Institut. Comparative Evaluation of the Sensitivity of SARSCoV-2 Antigen Rapid Tests.

https://www.pei.de/SharedDocs/Downloads/EN/newsroomen/dossiers/evaluation-sars-cov2-antigen-tests-overview-04-122020.pdf?_blob=publicationFile\&v=42

5) 5) Instand. Quantitative Bezugsproben zur Verbesserung der Vergleichbarkeit und Bewertung von Laborergebnissen zum VirusgenomNachweis von SARS-CoV-2.

6) World Health Organization. Antigen-detection in the diagnosis of SARSCoV-2 infection using rapid immunoassays. 11. 09. 2020. Interim guidance. WHO-2019-nCoV-Antigen_Detection-2020.1-eng\%20(5).pdf

7) US FDA. https://www.fda.gov/medical-devices/coronavirus-disease-2019covid-19-emergency-use-authorizations-medical-devices/in-vitrodiagnostics-euas\#individual-antigen

8) ECDC. Options for the use of rapid antigen tests for COVID-19 in the EU/EEA and the UK. 19 November 2020. Technical report. https://www.ecdc.europa.eu/sites/default/files/documents/Options-use-ofrapid-antigen-tests-for-COVID19-19-11-2020.pdf

9) Schweizerischer Bundesrat. SR 818.101.24 - Verordnung 3 vom 19. Juni 2020 über Massnahmen zur Bekämpfung des Coronavirus (Covid-19) 
(Covid-19-Verordnung 3), Anhang 5a.

https://www.fedlex.admin.ch/eli/cc/2020/438/de\#annex_5_a

10) Foundation for Innovative New Diagnostics (FIND).

https://www.finddx.org/sarscov2-eval-antigen/

11) Krüger LJ, Gaeddert M, Köppel L, Brümmer LE, Gottschalk C, Miranda IB, Schnitzler P, Kräusslich HG, Lindner AK, Nikolai O, Mockenhaupt FP, Seybold J, Corman VM, Drosten C, Pollock NR, Cubas-Atienzar AI, Kontogianni K, Collins A, Wright AH, Knorr B, Welker A, de Vos M, Sacks JA, Adams ER, Denkinger CM. Evaluation of the accuracy, ease of use and limit of detection of novel, rapid, antigen-detecting point-of-care diagnostics for SARS-CoV-2.

doi: https://doi.org/10.1101/2020.10.01.20203836 medRxiv preprint

12) Young S, Taylor SN, Cammarata CL, Roger-Dalbert C, Montano A, GriegoFullbright C, Burgard C, Fernandez C, Eckert K, Andrews JC, Ren H, Allen J, Ackerman R, Cooper CK. Clinical evaluation of BD VeritorTM SARSCoV-2 point-of-care test performance compared to PCR-based testing and versus the Sofia ${ }^{\circledR} 2$ SARS Antigen point-of-care test.

doi: https://doi.org/10.1101/2020.09.01.20185777 medRxiv preprint

13) Weitzel T, Legarraga $P$, Iruretagoyena M, Pizarro G, Vollrath V, Arao R, Munita CM, Porte L. Head-to-head comparison of four antigen-based rapid detection tests for the diagnosis of 1 SARS-CoV-2 in respiratory samples https://doi.org/10.1101/2020.05.27.119255 doi: bioRxiv preprint

14) Corman VM, Haage VC, Bleicker T, Schmidt ML, Mühlemann B, Zuchowski M, Karen K, Lei J, Tscheak P, Möncke-Buchner E, Müller MA, Krumbholz A, Drexler JF, Drosten C. Comparison of seven commercial SARS-CoV-2 rapid Point-of-Care Antigen tests medRxiv preprint doi: https://doi.org/10.1101/2020.11.12.20230292

15) Berger A, Ngo Nsoga MT, Perez-Rodriguez JF, YA Aad, Sattonnet-Roche P, Gayet-Ageron A, Jaksic C, Torriani G, Boehm E, Kronig I, Sacks JA, de Vos M, Jacquerioz Bausch F, Chappuis F, Kaiser L, Schibler M, Eckerle I. Diagnostic accuracy of two commercial SARS-CoV-2 Antigen-detecting 1 rapid tests at the point of care in community-based testing centers medRxiv preprint doi: https://doi.org/10.1101/2020.11.20.20235341 
16) Eidgenössisches Departement des Innern EDI, Bundesamt für Gesundheit BAG, Taskforce BAG Covid-19 AG Testung, Validierte SARS-CoV-2-

Schnelltests nach diagnostischem Standard zur Fachanwendung

https://www.bag.admin.ch/bag/de/home/medizin-und-

forschung/heilmittel/covid-testung.html

17) Dinnes J, Deeks JJ, Adriano A, Berhane S, Davenport C, Dittrich S, Emperador D, Takwoingi Y, Cunningham J, Beese S, Dretzke J, Ferrante di Ru\&ano L, Harris IM, Price MJ, Taylor-Phillips S, Hoo- L, Leeflang MMG, Spijker R, Van den Bruel A.Rapid, point-of-care antigen and molecularbased tests for diagnosis of SARS-CoV-2 infection.Cochrane Database of Systematic Reviews 2020, Issue 8. Art. No.: CD013705.

DOI: 10.1002/14651858.CD013705. 
Table 1

Comparative evaluation results of SARS-CoV-2 antigen RDT passing the sensitivity criteria

(in alphabetical order of manufacturers)

\begin{tabular}{ll} 
No. & Manufacturer \\
\hline $\mathbf{1}$ & Abbott Rapid Diagnostics Jena GmbH \\
$\mathbf{2}$ & ACON Biotech (Hangzhou) Co., Ltd \\
$\mathbf{3}$ & Aesku Diagnostics GmbH \\
$\mathbf{4}$ & Affimedix \\
$\mathbf{5}$ & Amazing Biotech (Shanghai) Co., Ltd \\
$\mathbf{6}$ & Ameda Labordiagnostik GmbH \\
$\mathbf{7}$ & AmonMed (Xiamen) Biotechnology Co., Ltd. \\
$\mathbf{8}$ & Anbio (Xiamen) Biotechnology Co., Ltd \\
$\mathbf{9}$ & Anhui Deepblue Medical Technology Co., , Ltd. \\
$\mathbf{1 0}$ & ASAN PHARM.CO.,LTD. \\
$\mathbf{1 1}$ & Atlas Link Technology Co.,Ltd. \\
$\mathbf{1 2}$ & Avalun \\
$\mathbf{1 3}$ & AXIOM Gesellschaft für Diagnostica und Biochemica mbH \\
$\mathbf{1 4}$ & Azure Biotech Inc. \\
$\mathbf{1 5}$ & Becton Dickinson \\
$\mathbf{1 6}$ & Beijing Beier Bioengineering Co., Ltd. \\
$\mathbf{1 7}$ & Beijing Hotgen Biotech Co., Ltd. \\
$\mathbf{1 8}$ & Beijing Lepu Medical Technology Co., Ltd \\
$\mathbf{1 9}$ & Beijing Tigsun Diagnostics Co.;Ltd. \\
$\mathbf{2 0}$ & BIOMERICA Inc. \\
$\mathbf{2 1}$ & BIONOTE \\
$\mathbf{2 2}$ & BioRepair GmbH \\
$\mathbf{2 3}$ & BIOSYNEX SWISS SA \\
$\mathbf{2 4}$ & BTNX, Inc. (Biotrend Chemikalien Gmbh) \\
$\mathbf{2 5}$ & Chil Tibbi Mal. San. Tic. Ltd. Şti \\
$\mathbf{2 6}$ & Core Technology Co., Ltd. \\
$\mathbf{2 7}$ & DNA Diagnostic A/S. \\
$\mathbf{2 8}$ & Edinburgh Genetics Limited \\
$\mathbf{2 9}$ & Eurobio Scientific \\
$\mathbf{3 0}$ & Fujirebio Inc. (Mast Diagnostica GmbH) \\
$\mathbf{3 1}$ & Genrui Biotech Inc. \\
$\mathbf{3 2}$ & GenSure Biotech Inc. \\
$\mathbf{3 3}$ & Getein Biotech, Inc. \\
$\mathbf{3 4}$ & Green Cross Medical Science Corp. (Weko Pharma GmbH) \\
$\mathbf{3 5}$ & Guangdong Hecin Scientific,Inc. \\
$\mathbf{3 6}$ & Guangdong Wesail Biotech Co., Ltd. \\
$\mathbf{3 7}$ & Guangzhou Wondfo Biotech Co. Ltd \\
$\mathbf{3 8}$ & Hangzhou Clongene Biotech Co., Ltd. \\
$\mathbf{3 9}$ & Hangzhou Immuno Biotech Co.,Ltd. \\
$\mathbf{4 0}$ & Hangzhou Laihe Biotech Co., Ltd. (Lissner Qi GmbH) \\
$\mathbf{4 1}$ & Hangzhou Lysun Biotechnology Co., Ltd. \\
$\mathbf{4 2}$ & Hangzhou Testsea Biotechnology Co., Ltd \\
&
\end{tabular}

\section{Test name}

Sensitivity

Panbio ${ }^{\mathrm{TM}} \mathrm{COVID}-19$ Ag Rapid Test Device (NASOPHARYNGEAL)

\begin{tabular}{rrrr}
\hline $\mathbf{C T}<\mathbf{2 5}$ & $\mathbf{C T} \mathbf{2 5 - 3 0}$ & \multicolumn{1}{c}{$\mathbf{C T} \mathbf{>} \mathbf{3 0}$} & $\mathbf{C T} \mathbf{1 7 - 3 6}$ \\
\hline $100,0 \%$ & $60,9 \%$ & $0,0 \%$ & $64,0 \%$ \\
$94,1 \%$ & $4,3 \%$ & $0,0 \%$ & $34,0 \%$ \\
$82,4 \%$ & $17,4 \%$ & $0,0 \%$ & $36,0 \%$ \\
$100,0 \%$ & $47,8 \%$ & $0,0 \%$ & $58,0 \%$ \\
$76,5 \%$ & $8,7 \%$ & $0,0 \%$ & $30,0 \%$ \\
$100,0 \%$ & $78,3 \%$ & $0,0 \%$ & $70,0 \%$ \\
$100,0 \%$ & $87,0 \%$ & $30,0 \%$ & $80,0 \%$ \\
$100,0 \%$ & $52,2 \%$ & $0,0 \%$ & $58,0 \%$ \\
$100,0 \%$ & $39,1 \%$ & $0,0 \%$ & $52,0 \%$ \\
$100,0 \%$ & $69,6 \%$ & $0,0 \%$ & $66,0 \%$ \\
$100,0 \%$ & $60,9 \%$ & $0,0 \%$ & $62,0 \%$ \\
$94,1 \%$ & $13,0 \%$ & $0,0 \%$ & $38,0 \%$ \\
$100,0 \%$ & $52,2 \%$ & $0,0 \%$ & $58,0 \%$ \\
$76,5 \%$ & $13,0 \%$ & $20,0 \%$ & $36,0 \%$ \\
$83,3 \%$ & $8,7 \%$ & $0,0 \%$ & $34,0 \%$ \\
$77,8 \%$ & $0,0 \%$ & $0,0 \%$ & $28,0 \%$ \\
$100,0 \%$ & $47,8 \%$ & $0,0 \%$ & $56,0 \%$ \\
$100,0 \%$ & $26,1 \%$ & $0,0 \%$ & $46,0 \%$ \\
$100,0 \%$ & $87,0 \%$ & $30,0 \%$ & $80,0 \%$ \\
$100,0 \%$ & $30,4 \%$ & $0,0 \%$ & $48,0 \%$ \\
$100,0 \%$ & $65,2 \%$ & $0,0 \%$ & $66,0 \%$ \\
$100,0 \%$ & $78,3 \%$ & $0,0 \%$ & $70,0 \%$ \\
$100,0 \%$ & $78,3 \%$ & $11,1 \%$ & $74,0 \%$ \\
$94,1 \%$ & $13,0 \%$ & $10,0 \%$ & $40,0 \%$ \\
$100,0 \%$ & $60,9 \%$ & $0,0 \%$ & $62,0 \%$ \\
$88,2 \%$ & $26,1 \%$ & $0,0 \%$ & $42,0 \%$ \\
$100,0 \%$ & $39,1 \%$ & $10,0 \%$ & $54,0 \%$ \\
$100,0 \%$ & $34,8 \%$ & $0,0 \%$ & $50,0 \%$ \\
$94,1 \%$ & $34,8 \%$ & $0,0 \%$ & $48,0 \%$ \\
$100,0 \%$ & $21,7 \%$ & $0,0 \%$ & $46,0 \%$ \\
$94,1 \%$ & $56,5 \%$ & $0,0 \%$ & $58,0 \%$ \\
$94,1 \%$ & $13,0 \%$ & $0,0 \%$ & $38,0 \%$ \\
$100,0 \%$ & $82,6 \%$ & $0,0 \%$ & $72,0 \%$ \\
$83,3 \%$ & $8,7 \%$ & $0,0 \%$ & $34,0 \%$ \\
$82,4 \%$ & $13,0 \%$ & $0,0 \%$ & $34,0 \%$ \\
$100,0 \%$ & $52,2 \%$ & $11,1 \%$ & $62,0 \%$ \\
$88,2 \%$ & $0,0 \%$ & $0,0 \%$ & $30,0 \%$ \\
$94,4 \%$ & $34,8 \%$ & $0,0 \%$ & $50,0 \%$ \\
$88,2 \%$ & $13,0 \%$ & $0,0 \%$ & $36,0 \%$ \\
$94,4 \%$ & $17,4 \%$ & $0,0 \%$ & $42,0 \%$ \\
$100,0 \%$ & $78,3 \%$ & $0,0 \%$ & $70,0 \%$ \\
$100,0 \%$ & $47,8 \%$ & $0,0 \%$ & $56,0 \%$ \\
& & &
\end{tabular}

\begin{tabular}{rrrr}
\hline $\mathbf{C T}<\mathbf{2 5}$ & $\mathbf{C T} \mathbf{2 5 - 3 0}$ & \multicolumn{1}{c}{$\mathbf{C T} \mathbf{>} \mathbf{3 0}$} & $\mathbf{C T} \mathbf{1 7 - 3 6}$ \\
\hline $100,0 \%$ & $60,9 \%$ & $0,0 \%$ & $64,0 \%$ \\
$94,1 \%$ & $4,3 \%$ & $0,0 \%$ & $34,0 \%$ \\
$82,4 \%$ & $17,4 \%$ & $0,0 \%$ & $36,0 \%$ \\
$100,0 \%$ & $47,8 \%$ & $0,0 \%$ & $58,0 \%$ \\
$76,5 \%$ & $8,7 \%$ & $0,0 \%$ & $30,0 \%$ \\
$100,0 \%$ & $78,3 \%$ & $0,0 \%$ & $70,0 \%$ \\
$100,0 \%$ & $87,0 \%$ & $30,0 \%$ & $80,0 \%$ \\
$100,0 \%$ & $52,2 \%$ & $0,0 \%$ & $58,0 \%$ \\
$100,0 \%$ & $39,1 \%$ & $0,0 \%$ & $52,0 \%$ \\
$100,0 \%$ & $69,6 \%$ & $0,0 \%$ & $66,0 \%$ \\
$100,0 \%$ & $60,9 \%$ & $0,0 \%$ & $62,0 \%$ \\
$94,1 \%$ & $13,0 \%$ & $0,0 \%$ & $38,0 \%$ \\
$100,0 \%$ & $52,2 \%$ & $0,0 \%$ & $58,0 \%$ \\
$76,5 \%$ & $13,0 \%$ & $20,0 \%$ & $36,0 \%$ \\
$83,3 \%$ & $8,7 \%$ & $0,0 \%$ & $34,0 \%$ \\
$77,8 \%$ & $0,0 \%$ & $0,0 \%$ & $28,0 \%$ \\
$100,0 \%$ & $47,8 \%$ & $0,0 \%$ & $56,0 \%$ \\
$100,0 \%$ & $26,1 \%$ & $0,0 \%$ & $46,0 \%$ \\
$100,0 \%$ & $87,0 \%$ & $30,0 \%$ & $80,0 \%$ \\
$100,0 \%$ & $30,4 \%$ & $0,0 \%$ & $48,0 \%$ \\
$100,0 \%$ & $65,2 \%$ & $0,0 \%$ & $66,0 \%$ \\
$100,0 \%$ & $78,3 \%$ & $0,0 \%$ & $70,0 \%$ \\
$100,0 \%$ & $78,3 \%$ & $11,1 \%$ & $74,0 \%$ \\
$94,1 \%$ & $13,0 \%$ & $10,0 \%$ & $40,0 \%$ \\
$100,0 \%$ & $60,9 \%$ & $0,0 \%$ & $62,0 \%$ \\
$88,2 \%$ & $26,1 \%$ & $0,0 \%$ & $42,0 \%$ \\
$100,0 \%$ & $39,1 \%$ & $10,0 \%$ & $54,0 \%$ \\
$100,0 \%$ & $34,8 \%$ & $0,0 \%$ & $50,0 \%$ \\
$94,1 \%$ & $34,8 \%$ & $0,0 \%$ & $48,0 \%$ \\
$100,0 \%$ & $21,7 \%$ & $0,0 \%$ & $46,0 \%$ \\
$94,1 \%$ & $56,5 \%$ & $0,0 \%$ & $58,0 \%$ \\
$94,1 \%$ & $13,0 \%$ & $0,0 \%$ & $38,0 \%$ \\
$100,0 \%$ & $82,6 \%$ & $0,0 \%$ & $72,0 \%$ \\
$83,3 \%$ & $8,7 \%$ & $0,0 \%$ & $34,0 \%$ \\
$82,4 \%$ & $13,0 \%$ & $0,0 \%$ & $34,0 \%$ \\
$100,0 \%$ & $52,2 \%$ & $11,1 \%$ & $62,0 \%$ \\
$88,2 \%$ & $0,0 \%$ & $0,0 \%$ & $30,0 \%$ \\
$94,4 \%$ & $34,8 \%$ & $0,0 \%$ & $50,0 \%$ \\
$88,2 \%$ & $13,0 \%$ & $0,0 \%$ & $36,0 \%$ \\
$94,4 \%$ & $17,4 \%$ & $0,0 \%$ & $42,0 \%$ \\
$100,0 \%$ & $78,3 \%$ & $0,0 \%$ & $70,0 \%$ \\
$100,0 \%$ & $47,8 \%$ & $0,0 \%$ & $56,0 \%$ \\
& & &
\end{tabular}

\begin{tabular}{rrrr}
\hline $\mathbf{C T}<\mathbf{2 5}$ & $\mathbf{C T} \mathbf{2 5 - 3 0}$ & \multicolumn{1}{c}{$\mathbf{C T} \mathbf{>} \mathbf{3 0}$} & $\mathbf{C T} \mathbf{1 7 - 3 6}$ \\
\hline $100,0 \%$ & $60,9 \%$ & $0,0 \%$ & $64,0 \%$ \\
$94,1 \%$ & $4,3 \%$ & $0,0 \%$ & $34,0 \%$ \\
$82,4 \%$ & $17,4 \%$ & $0,0 \%$ & $36,0 \%$ \\
$100,0 \%$ & $47,8 \%$ & $0,0 \%$ & $58,0 \%$ \\
$76,5 \%$ & $8,7 \%$ & $0,0 \%$ & $30,0 \%$ \\
$100,0 \%$ & $78,3 \%$ & $0,0 \%$ & $70,0 \%$ \\
$100,0 \%$ & $87,0 \%$ & $30,0 \%$ & $80,0 \%$ \\
$100,0 \%$ & $52,2 \%$ & $0,0 \%$ & $58,0 \%$ \\
$100,0 \%$ & $39,1 \%$ & $0,0 \%$ & $52,0 \%$ \\
$100,0 \%$ & $69,6 \%$ & $0,0 \%$ & $66,0 \%$ \\
$100,0 \%$ & $60,9 \%$ & $0,0 \%$ & $62,0 \%$ \\
$94,1 \%$ & $13,0 \%$ & $0,0 \%$ & $38,0 \%$ \\
$100,0 \%$ & $52,2 \%$ & $0,0 \%$ & $58,0 \%$ \\
$76,5 \%$ & $13,0 \%$ & $20,0 \%$ & $36,0 \%$ \\
$83,3 \%$ & $8,7 \%$ & $0,0 \%$ & $34,0 \%$ \\
$77,8 \%$ & $0,0 \%$ & $0,0 \%$ & $28,0 \%$ \\
$100,0 \%$ & $47,8 \%$ & $0,0 \%$ & $56,0 \%$ \\
$100,0 \%$ & $26,1 \%$ & $0,0 \%$ & $46,0 \%$ \\
$100,0 \%$ & $87,0 \%$ & $30,0 \%$ & $80,0 \%$ \\
$100,0 \%$ & $30,4 \%$ & $0,0 \%$ & $48,0 \%$ \\
$100,0 \%$ & $65,2 \%$ & $0,0 \%$ & $66,0 \%$ \\
$100,0 \%$ & $78,3 \%$ & $0,0 \%$ & $70,0 \%$ \\
$100,0 \%$ & $78,3 \%$ & $11,1 \%$ & $74,0 \%$ \\
$94,1 \%$ & $13,0 \%$ & $10,0 \%$ & $40,0 \%$ \\
$100,0 \%$ & $60,9 \%$ & $0,0 \%$ & $62,0 \%$ \\
$88,2 \%$ & $26,1 \%$ & $0,0 \%$ & $42,0 \%$ \\
$100,0 \%$ & $39,1 \%$ & $10,0 \%$ & $54,0 \%$ \\
$100,0 \%$ & $34,8 \%$ & $0,0 \%$ & $50,0 \%$ \\
$94,1 \%$ & $34,8 \%$ & $0,0 \%$ & $48,0 \%$ \\
$100,0 \%$ & $21,7 \%$ & $0,0 \%$ & $46,0 \%$ \\
$94,1 \%$ & $56,5 \%$ & $0,0 \%$ & $58,0 \%$ \\
$94,1 \%$ & $13,0 \%$ & $0,0 \%$ & $38,0 \%$ \\
$100,0 \%$ & $82,6 \%$ & $0,0 \%$ & $72,0 \%$ \\
$83,3 \%$ & $8,7 \%$ & $0,0 \%$ & $34,0 \%$ \\
$82,4 \%$ & $13,0 \%$ & $0,0 \%$ & $34,0 \%$ \\
$100,0 \%$ & $52,2 \%$ & $11,1 \%$ & $62,0 \%$ \\
$88,2 \%$ & $0,0 \%$ & $0,0 \%$ & $30,0 \%$ \\
$94,4 \%$ & $34,8 \%$ & $0,0 \%$ & $50,0 \%$ \\
$88,2 \%$ & $13,0 \%$ & $0,0 \%$ & $36,0 \%$ \\
$94,4 \%$ & $17,4 \%$ & $0,0 \%$ & $42,0 \%$ \\
$100,0 \%$ & $78,3 \%$ & $0,0 \%$ & $70,0 \%$ \\
$100,0 \%$ & $47,8 \%$ & $0,0 \%$ & $56,0 \%$ \\
& & &
\end{tabular}

Flowflex SARS-CoV-2-Antigenschnelltest (Nasopharynxtupfer)

Aesku Rapid SARS-CoV-2 Rapid Test

TestNOW® - COVID-19 Antigen

CoroVisio Covid-19 Ag Versieglungsröhrchen Teststreifen (Kolloidales Gold)

\begin{tabular}{rrrr}
\hline $\mathbf{C T}<\mathbf{2 5}$ & $\mathbf{C T} \mathbf{2 5 - 3 0}$ & \multicolumn{1}{c}{$\mathbf{C T} \mathbf{>} \mathbf{3 0}$} & $\mathbf{C T} \mathbf{1 7 - 3 6}$ \\
\hline $100,0 \%$ & $60,9 \%$ & $0,0 \%$ & $64,0 \%$ \\
$94,1 \%$ & $4,3 \%$ & $0,0 \%$ & $34,0 \%$ \\
$82,4 \%$ & $17,4 \%$ & $0,0 \%$ & $36,0 \%$ \\
$100,0 \%$ & $47,8 \%$ & $0,0 \%$ & $58,0 \%$ \\
$76,5 \%$ & $8,7 \%$ & $0,0 \%$ & $30,0 \%$ \\
$100,0 \%$ & $78,3 \%$ & $0,0 \%$ & $70,0 \%$ \\
$100,0 \%$ & $87,0 \%$ & $30,0 \%$ & $80,0 \%$ \\
$100,0 \%$ & $52,2 \%$ & $0,0 \%$ & $58,0 \%$ \\
$100,0 \%$ & $39,1 \%$ & $0,0 \%$ & $52,0 \%$ \\
$100,0 \%$ & $69,6 \%$ & $0,0 \%$ & $66,0 \%$ \\
$100,0 \%$ & $60,9 \%$ & $0,0 \%$ & $62,0 \%$ \\
$94,1 \%$ & $13,0 \%$ & $0,0 \%$ & $38,0 \%$ \\
$100,0 \%$ & $52,2 \%$ & $0,0 \%$ & $58,0 \%$ \\
$76,5 \%$ & $13,0 \%$ & $20,0 \%$ & $36,0 \%$ \\
$83,3 \%$ & $8,7 \%$ & $0,0 \%$ & $34,0 \%$ \\
$77,8 \%$ & $0,0 \%$ & $0,0 \%$ & $28,0 \%$ \\
$100,0 \%$ & $47,8 \%$ & $0,0 \%$ & $56,0 \%$ \\
$100,0 \%$ & $26,1 \%$ & $0,0 \%$ & $46,0 \%$ \\
$100,0 \%$ & $87,0 \%$ & $30,0 \%$ & $80,0 \%$ \\
$100,0 \%$ & $30,4 \%$ & $0,0 \%$ & $48,0 \%$ \\
$100,0 \%$ & $65,2 \%$ & $0,0 \%$ & $66,0 \%$ \\
$100,0 \%$ & $78,3 \%$ & $0,0 \%$ & $70,0 \%$ \\
$100,0 \%$ & $78,3 \%$ & $11,1 \%$ & $74,0 \%$ \\
$94,1 \%$ & $13,0 \%$ & $10,0 \%$ & $40,0 \%$ \\
$100,0 \%$ & $60,9 \%$ & $0,0 \%$ & $62,0 \%$ \\
$88,2 \%$ & $26,1 \%$ & $0,0 \%$ & $42,0 \%$ \\
$100,0 \%$ & $39,1 \%$ & $10,0 \%$ & $54,0 \%$ \\
$100,0 \%$ & $34,8 \%$ & $0,0 \%$ & $50,0 \%$ \\
$94,1 \%$ & $34,8 \%$ & $0,0 \%$ & $48,0 \%$ \\
$100,0 \%$ & $21,7 \%$ & $0,0 \%$ & $46,0 \%$ \\
$94,1 \%$ & $56,5 \%$ & $0,0 \%$ & $58,0 \%$ \\
$94,1 \%$ & $13,0 \%$ & $0,0 \%$ & $38,0 \%$ \\
$100,0 \%$ & $82,6 \%$ & $0,0 \%$ & $72,0 \%$ \\
$83,3 \%$ & $8,7 \%$ & $0,0 \%$ & $34,0 \%$ \\
$82,4 \%$ & $13,0 \%$ & $0,0 \%$ & $34,0 \%$ \\
$100,0 \%$ & $52,2 \%$ & $11,1 \%$ & $62,0 \%$ \\
$88,2 \%$ & $0,0 \%$ & $0,0 \%$ & $30,0 \%$ \\
$94,4 \%$ & $34,8 \%$ & $0,0 \%$ & $50,0 \%$ \\
$88,2 \%$ & $13,0 \%$ & $0,0 \%$ & $36,0 \%$ \\
$94,4 \%$ & $17,4 \%$ & $0,0 \%$ & $42,0 \%$ \\
$100,0 \%$ & $78,3 \%$ & $0,0 \%$ & $70,0 \%$ \\
$100,0 \%$ & $47,8 \%$ & $0,0 \%$ & $56,0 \%$ \\
& & &
\end{tabular}

AMP Rapid Test SARS-CoV-2 Ag

COVID-19 Antigen Rapid Test Kit (Colloidal Gold)

Rapid Covid-19 Antigen Test (Colloidal Gold)

COVID-19 (SARS CoV-2) Antigen Test Kit (Colloidal Gold)

Asan Easy Test COVID-19 Ag

Nova Test SARS-CoV-2 Antigen Rapid Test Kit

Ksmart@ SARS-COV2 Antigen Rapid Test

Axiom Diagnostics COVID-19 Ag Schnelltest

Dia Sure Covid-19 Antigen Rapid Test Device (Nasopharyngeal/Oropharyngeal Swab)

BD Veritor ${ }^{\mathrm{TM}}$ System for Rapid Detection of SARS-CoV-2

Covid-19 Antigen Schnelltest

Novel Coronavirus 2019-nCoV Antigen Test (Colloidal gold)

SARS-CoV-2 Antigen Rapid Test Kit

Tigsun COVID-19 Saliva Antigen Rapid Test

COVID-19-Antigen-Schnelltest (Nasopharyngeal-Abstrich)

NowCheck® COVID-19 Ag Test

Covid 19 Antigen Schnelltest

BIOSYNEX COVID-19 Ag BSS

Rapid Response COVID-19 Rapid Test Device

COVID-19 Antigen Schnell Test (Nasopharyngeal / Oropharyngeal Tupfer Kassette)

Canea COVID-19 Antigen Schnelltest

Covid-19 Antigen Detection Kit

Edinburgh Genetics ActivXpress+ COVID-19 Antigen Complete Testing Kit

EBS SARS-CoV-2 Ag Rapid Test

ESPLINE $\circledast$ SARS-CoV-2

Genrui SARS-CoV-2 Antigen Test Kit (Colloidal Gold)

DIA-COVID $\circledast$ COVID-19 Ag Rapid Test Kit

One Step Test for SARS-CoV-2 Antigen (Colloidal Gold)

Genedia W Covid-19 Ag

2019-nCoV Antigen Test Kit(colloidal gold method)

COVID-19 Ag Test Kit

Wondfo SARS-CoV-2 Antigen Test (Lateral Flow Method)

Clungene COVID-19 Antigen Rapid Test

IMMUNOBIO SARS-CoV-2 Antigen-Schnelltest (COVID-19 Ag)

Lyher Novel Coronavirus (COVID-19) Antigen Test Kit (Colloidal Gold)

Lysun COVID-19 Antigen Rapid Test Device (Colloidal Gold)

Testsealabs® Rapid Test Kit COVID-19 Antigen Test Cassette 


CT 25-30

$0,0 \%$

CT 17-36

45 Jiangsu Diagnostics Biotechnology Co., Ltd

COVID-19 Antigen Rapid Test Cassette (Colloidal Gold)

$94,4 \%$

$26,1 \%$

SARS-CoV-2-Antigen-Testkit (LFIA)

47 Joinstar Biomedical Technology Co., Ltd (CIV care impuls Vertrieb) CoVID-19 Antigen Schnelltest (Colloidal Gold)

48 Labnovation Technologies, Inc.

49 Lumigenex (Suzhou) Co., Ltd.

50 LumiQuick Diagnostics, Inc.

51 LumiraDX

52 MEDsan GmbH

53 Merlin Biomedical (Xiamen) Co., Ltd.

54 Mölab GmbH

55 MP Biomedicals Germany GmbH

56 nal von minden gmbh

57 Nanjing Norman Biological Technology Co.,Ltd

58 NanoEntek Inc

59 Nantong Diagnos Biotechnology Co., Ltd

60 New Gene (Hangzhou) Bioengineering Co., Ltd.

61 Novatech Tibbi Cihaz Ürünleri San. Ve Tic.A.S.

62 Oncosem Onkolojik Sistemler San. Ve Tic. A.S.

63 PCL, Inc.

64 PerGrande BioTech Development Co., Ltd.

65 Precision Biosensor Inc. (Axon Lab AG)

66 ProGnosis Biotech

67 Quidel Corporation

68 Qingdao Hightop Biotech Co., Ltd.

69 R-Biopharm AG

70 Safecare Biotech Hangzhou Co., Ltd.

71 Salofa OY

72 ScheBo Biotech AG

73 SD BIOSENSOR (Roche Diagnostics $\mathrm{GmbH}$ )

74 SD BIOSENSOR

75 SD BIOSENSOR

76 SGA Mühendislik DAN. EG. Icve DIS.Ltd.STI

77 Shenzhen Lvshiyuan Biotechnology Co., Ltd.

78 Shenzhen Microprofit Biotech Co., Ltd

79 Shenzhen Watmind Medical Co Ltd.

80 Shenzhen Watmind Medical Co.,Ltd.

81 Shenzhen Zhenrui Biotech co.Ltd.

82 Siemens Healthineers

83 Sugentech, Inc

84 Toda Pharma

85 Triplex International Biosciences (China) Co., Ltd.

86 ulti med Products (Deutschland) $\mathrm{GmbH}$

87 Vitrosens Biyoteknoloji Ltd. Sti

Labnovation SARS-CoV-2 Antigen Rapid Test Kit (Immunochromatography)

$94,1 \%$

$100,0 \%$

$94,1 \%$

PocRoc SARS-CoV-2, Antigen Schnelltest Set (Kolloidales Gold)

QuickProfile Covid-19 Antigen Test Card

LumiraDx SARS-CoV-2 Ag Test

MEDsan® SARS-CoV-2 Antigen Rapid Test

SARS-CoV-2 Antigen Rapid Test Cassette

mö-screen Corona Antigen Test

Rapid SARS-CoV-2 Antigen Test Card

NADAL $\odot$ COVID-19 Ag Schnelltest

Novel Coronavirus (2019-nCOV) Antigen Testing Kit (Colloidal Gold)

FREND ${ }^{\text {TM }}$ COVID-19 Ag

COVID-19 Antigen Saliva Test Kit (Colloidal Gold)

Covid-19-Antigen-Testkit

novacheck®-Ag SARS-CoV-2 Covid-19 Antigen Rapid Test

CAT Antigen Covid Rapid Test

PCL COVID19 Ag Gold Saliva

SARS-CoV-2 Antigen Detection Kit (Colloidal Gold Immunochromatographic Assay)

Exdia COVID-19-Ag-Test

Rapid Test Ag 2019-nCoV

Sofia SARS Antigen FIA

Hightop SARS-CoV-2 (Covid-19) Antigen Rapid Test

RIDA®QUICK SARS-CoV-2 Antigen

Safecare COVID-19 Ag Rapid Test Kit (Swab)

salocor SARS-CoV-2 Antigen Rapid Test Cassette (Nasopharyngeal swab)

ScheBo SARS-CoV-2 Quick Antigen

SARS-CoV-2 Rapid Antigen Test

STANDARD TM Q COVID-19 Ag Test

STANDARD TM F COVID-19 Ag FIA

V-Chek SARS-CoV-2 Rapid Ag Test Kit (Colloidal Gold)

Green Spring SARS-CoV-2 Antigen Rapid Test Kit (Colloidal Gold)

fluorecare COVID-19 SARS-CoV-2 Spike Protein Test Kit (Colloidal Gold Chromatographic Immunoassay)

$\begin{array}{ll}78,3 \% & 0,0 \% \\ 21,7 \% & 0,0 \%\end{array}$

$46,0 \%$

SARS-CoV-2 Ag Diagnostic Test Kit (Colloidal Gold)

SARS-CoV-2 Ag Diagnostic Test Kit (Immuno-fluorescence)

Zhenrui COVID-19 (SARS-COV-2) Antigen Test Kits

CLINITEST $₫$ Rapid COVID-19 Antigen Tes

SGTi-flex COVID-19 Ag

Toda Coronadiag Ag

SARS-CoV-2 Antigen Rapid Test Kit

COVID-19 Antigen Speicheltest (Immunochromatographie)

RapidFor SARS-CoV-2 Rapid Antigen Test Colloidal Gold

$\begin{array}{ll}21,7 \% & 0,0 \% \\ 60,9 \% & 0,0 \% \\ 17,4 \% & 0,0 \%\end{array}$

$42,0 \%$

\begin{tabular}{l}
$60,9 \%-64,0 \%$ \\
\hline $17,4 \%-0,0 \%-0 \%$
\end{tabular}

$0.0 \%$
$-60 \%$

$\begin{array}{llll}100,0 \% & 91,3 \% & 20,0 \% & 80,0 \%\end{array}$

$\begin{array}{llll}100,0 \% & 52,2 \% & 0,0 \% & 60,0 \%\end{array}$

$100,0 \%-47,8 \% \quad 0,0 \% \quad 58,0 \%$

$\begin{array}{llll}100,0 \% & 82,6 \% & 0,0 \% & 72,0 \%\end{array}$

$\begin{array}{llll}100,0 \% & 47,8 \% & 0,0 \% & 58,0 \%\end{array}$

$100,0 \% \quad 43,5 \% \quad 0,0 \% \quad 54,0 \%$

$\begin{array}{llll}83,3 \% & 13,0 \% & 0,0 \% & 36,0 \% \\ 94,1 \% & 26,1 \% & 0,0 \% & 44,0 \%\end{array}$

$88,2 \%-8,7 \% \quad 0.0 \% \quad 34,0 \%$

$\begin{array}{llll}100,0 \% & 56,5 \% & 0,0 \% & 60,0 \%\end{array}$

$\begin{array}{llll}100,0 \% & 87,0 \% & 20,0 \% & 78,0 \%\end{array}$

$\begin{array}{llll}94,1 \% & 21,7 \% & 0,0 \% & 42,0 \%\end{array}$

$\begin{array}{rlll}94,1 \% & 30,4 \% & 0,0 \% & 46,0 \%\end{array}$

$\begin{array}{llll}100,0 \% & 52,2 \% & 0,0 \% & 58,0 \% \\ 100,0 \% & 17,4 \% & 0,0 \% & 42,0 \%\end{array}$

$\begin{array}{llll}100,0 \% & 60,9 \% & 0,0 \% & 64,0 \%\end{array}$

$\begin{array}{llll}94,1 \% & 65,2 \% & 10,0 \% & 64,0 \%\end{array}$

$\begin{array}{llll}88,9 \% & 8,7 \% & 0,0 \% & 36,0 \%\end{array}$

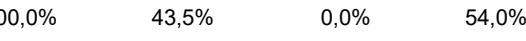

$\begin{array}{llll}100,0 \% & 17,4 \% & 0,0 \% & 44,0 \%\end{array}$

$\begin{array}{llll}100,0 \% & 60,9 \% & 0,0 \% & 62,0 \%\end{array}$

$\begin{array}{llll}82,4 \% & 13,0 \% & 0,0 \% & 34,0 \%\end{array}$

$\begin{array}{llll}100,0 \% & 91,3 \% & 10,0 \% & 78,0 \%\end{array}$

$\begin{array}{llll}80,9 \% & 30,4 \% & 0,0 \% & 46,0 \%\end{array}$

$\begin{array}{llll}88,9 \% & 30,4 \% & 0,0 \% & 46,0 \%\end{array}$

$\begin{array}{llll}100,0 \% & 65,2 \% & 0,0 \% & 66,0 \%\end{array}$

$\begin{array}{rrrr}94,1 \% & 26,1 \% & 0,0 \% & 44,0 \% \\ 100,0 \% & 95,7 \% & 40,0 \% & 86,0 \%\end{array}$

$\begin{array}{llll}100,0 \% & 47,8 \% & 10,0 \% & 58,0 \%\end{array}$

$\begin{array}{llll}100,0 \% & 95,7 \% & 20,0 \% & 82,0 \%\end{array}$

$\begin{array}{llll}100,0 \% & 60,9 \% & 0,0 \% & 62,0 \%\end{array}$

$\begin{array}{llll}82,4 \% & 13,0 \% & 0,0 \% & 34,0 \%\end{array}$

$\begin{array}{rrrr}100,0 \% & 87,0 \% & 0,0 \% & 34,0 \% \\ 100 \% & 73,9 \% & 0,0 \% & 68,0 \%\end{array}$

$\begin{array}{llll}100,0 \% & 73,9 \% & 0,0 \% & 68,0 \%\end{array}$

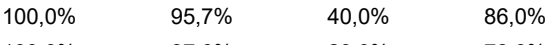

$\begin{array}{llll}100,0 \% & 87,0 \% & 20,0 \% & 78,0 \%\end{array}$

$\begin{array}{llll}100,0 \% & 95,7 \% & 20,0 \% & 82,0 \%\end{array}$

$\begin{array}{rrrr}100,0 \% & 30,4 \% & 0,0 \% & 48,0 \%\end{array}$ 
No. Manufacturer

Test name

Wantai (Beijing Wantai Biological Pharmacy Enterprise Co., Ltd.) SARS-CoV-2 Ag Rapid Test (FIA)

Wuhan EasyDiagnosis Biomedicine Co., Ltd $\quad$ COVID-19 (SARS-CoV-2) Antigen Test Kit

Wuhan Life Origin Biotech Joint Stock Co., Ltd.

Wuhan UNscience Biotechnology Co., Ltd.

SARS-CoV-2 Antigen Assay Kit (Immunoct

SARS-CoV-2 An

Xiamen Boson Biotech Co., Ltd

Xiamen WIZ Biotech Co., Ltd.

Zet Medikal Tekstil Dis Ticaret Ltd. STI.

Zhejiang Anji Saianfu Biotech Co.,Ltd.

Wizbiotech SARS-CoV-2 Antigen Rapid Test

softec SARS COV-2 (Covid-19) Antigen Test Kit

reOpenTest COVID-19 Antigen Rapid Test (Colloidal Gold)

Coronavirus Ag Rapid Test Cassette (Swab)
Sensitivity

CT $<25$

$100,0 \%$

$100,0 \%$

T 25-30

T $>30$

$100,0 \%$

$78,3 \%$

$0,0 \%$

$\begin{array}{lll}56,5 \% & 0,0 \% & 60,0 \% \\ 17,4 \% & 0,0 \% & 38,0 \%\end{array}$

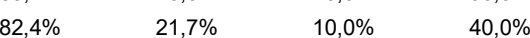

96 Zhejiang Orient Gene Biotech Co.,Ltd 
Table 2

Comparative evaluation results of SARS-CoV-2 antigen RDT missing the sensitivity criteria

(in alphabetical order of manufacturers)

\begin{tabular}{|c|c|c|c|c|c|c|}
\hline \multirow[b]{2}{*}{ No. } & \multirow[b]{2}{*}{ Manufacturer } & \multirow[b]{2}{*}{ Test name } & \multicolumn{4}{|c|}{ Sensitivity } \\
\hline & & & CT $<25$ & CT 25-30 & CT $>30$ & CT 17-36 \\
\hline 1 & Acro Biotech Inc & Acro COVID-19 Antigen Rapid Test & $16,7 \%$ & $0,0 \%$ & $0,0 \%$ & $6,0 \%$ \\
\hline 2 & Aikang Diagnostics Co., Ltd. & SARS-CoV-2 Antigen Test Kit (Immunochromatography) & $11,8 \%$ & $0,0 \%$ & $0,0 \%$ & $4,0 \%$ \\
\hline 3 & Beijing Savant Biotechnology Co., Ltd & New Coronavirus (SARS-CoV-2) N Protein Detection Kit (Fluorescence Immunchromatography) & $0,0 \%$ & $0,0 \%$ & $0,0 \%$ & $0,0 \%$ \\
\hline 4 & Certest Biotec S. L. & CerTest SARS-CoV-2 & $29,4 \%$ & $0,0 \%$ & $0,0 \%$ & $10,0 \%$ \\
\hline 5 & Coris Bioconcept & COVID-19 Ag Respi-Strip & $33,3 \%$ & $0,0 \%$ & $0,0 \%$ & $12,0 \%$ \\
\hline 6 & Hangzhou AllTest Biotech Co. Ltd. & COVID-19 AG AllTest & $16,7 \%$ & $0,0 \%$ & $0,0 \%$ & $6,0 \%$ \\
\hline 7 & Hangzhou Biotest Biotech Co., Ltd. & Lumiratek SARS-CoV-2 Antigen Rapid Test Cassette & $29,4 \%$ & $0,0 \%$ & $0,0 \%$ & $10,0 \%$ \\
\hline 8 & Hangzhou Genesis Biocontrol Co., Ltd & KaiBiLi COVID-19 Antigen Rapid Test Device & $52,9 \%$ & $0,0 \%$ & $0,0 \%$ & $18,0 \%$ \\
\hline 9 & Hangzhou Realy Tech Co., Ltd. & Novel Coronavirus (SARS-Cov-2) Antigen Rapid Test Cassette (swab) & $58,8 \%$ & $0,0 \%$ & $0,0 \%$ & $20,0 \%$ \\
\hline 10 & Inzek International Trading & Biozek medical COVID-19 Antigen Rapid Test Cassette & $52,9 \%$ & $0,0 \%$ & $0,0 \%$ & $18,0 \%$ \\
\hline 11 & Joinstar Biomedical Technology Co., Ltd & COVID-19 Antigen Rapid Test (Latex) & $0,0 \%$ & $0,0 \%$ & $0,0 \%$ & $0,0 \%$ \\
\hline 12 & Joysbio (Tianjin) Biotechnology Co., Ltd. & Joysbio SARS-CoV-2 Antigen Rapid Test Kit (Colloidal Gold) & $47,1 \%$ & $4,3 \%$ & $0,0 \%$ & $18,0 \%$ \\
\hline 13 & Lionex GmbH & Lionex COVID-19 Ag Rapid Test & $0,0 \%$ & $0,0 \%$ & $0,0 \%$ & $0,0 \%$ \\
\hline 14 & Medicon Co., Ltd. & Trueline COVID-19 Ag Rapid Test & $58,8 \%$ & $4,3 \%$ & $0,0 \%$ & $22,0 \%$ \\
\hline 15 & Mexacare GmbH Heidelberg & QuickTestCorona COVID-19 Antigen Schnelltest & $52,9 \%$ & $4,3 \%$ & $0,0 \%$ & $20,0 \%$ \\
\hline 16 & nal von minden $\mathrm{GmbH}$ & dedicio Medical Test COVID-19 Ag plus Test & $35,3 \%$ & $0,0 \%$ & $0,0 \%$ & $12,0 \%$ \\
\hline 17 & Rapigen & Biocredit COVID-19 Ag & $16,7 \%$ & $0,0 \%$ & $0,0 \%$ & $6,0 \%$ \\
\hline 18 & Servoprax & Cleartest Coronaantigen & $66,7 \%$ & $0,0 \%$ & $0,0 \%$ & $24,0 \%$ \\
\hline 19 & Spring Healthcare Services SP zoo & SARS-Cov-2 Antigen Rapid Test Cassette (swab) & $29,4 \%$ & $0,0 \%$ & $0,0 \%$ & $10,0 \%$ \\
\hline 20 & SureScreen Diagnostics Ltd & COVID-19 Antigen Rapid Test Cassette & $52,9 \%$ & $0,0 \%$ & $0,0 \%$ & $18,0 \%$ \\
\hline 21 & TaiDoc TechnologyCorp. & FORA COVID-19 ANTIGEN RAPID TEST & $27,8 \%$ & $0,0 \%$ & $0,0 \%$ & $10,0 \%$ \\
\hline 22 & Unioninvest & Unibioscience COVID-19 Rapid Antigen Test & $0,0 \%$ & $0,0 \%$ & $0,0 \%$ & $0,0 \%$ \\
\hline 23 & VivaChek Biotech (Hangzhou) Co,Ltd. & VivaDiag SARS-CoV-2 Ag Rapid Test & $50,0 \%$ & $0,0 \%$ & $0,0 \%$ & $18,0 \%$ \\
\hline 24 & VivaChek Biotech (Hangzhou) Co,Ltd. & VivaDiag Pro SARS-CoV-2 Ag Rapid Test & $64,7 \%$ & $0,0 \%$ & $0,0 \%$ & $22,0 \%$ \\
\hline 25 & W.H.P.M, Inc & First SIGN SARS-CoV-2 Antigen Test & $47,1 \%$ & $0,0 \%$ & $0,0 \%$ & $16,0 \%$ \\
\hline 26 & Xiamen Zhongsheng Langjie Biotechnology Co., Ltd & Covid-19 Antigen Test Cassette & $11,8 \%$ & $0,0 \%$ & $0,0 \%$ & $4,0 \%$ \\
\hline
\end{tabular}

